\title{
Modelling and analysis of CAD expert behaviour in using manual input devices
}

\author{
Halimahtun M. Khalid \\ Institute of Design and Ergonomics Application, Universiti Malaysia Sarawak, 94300 Kota Samarahan, Sarawak, Malaysia
}

Received 26 October 1999; accepted 2 August 2000

\begin{abstract}
In computer-aided design (CAD) systems the tablet-plus-keyboard has been the most common configuration of input devices. Their use in CAD work requires several additional subsidiary tasks of device manipulation, decision-making, perceptual matching and error correction. These tasks, imposed by the systems design, are mostly additional to the primary task of drawing. This observational study documented the nature of non-optimal behaviour in using manual input devices to perform CAD tasks by analysing behaviour protocol of designers at work recorded on video. A task analysis illustrated the variety of knowledge resources in CAD performance. The use of manual input devices resulted in substantial movement of the eyes between different parts of the system, thus reducing the time spent looking at the graphics screen for design activity; and substantial movement of the hand between input devices, thus incurring time. The imposition of subsidiary tasks is considered non-optimal since more resources were deployed to manipulate the input devices and correct errors than to perform mental operations required by the drawing task. A blackboard model of system behaviour illustrates individual differences in knowledge recruitment due to different tasks and systems used. The model, based on behaviour protocols, provides a novel approach to task analysis of CAD expert performance.
\end{abstract}

\section{Relevance to industry}

The use of CAD systems in manufacturing is commonplace. It is important that these systems are designed to meet CAD designers' needs and to support their task performance. The study has implications for the use of speech input devices in CAD which complement manual input and enhance the overall productivity of CAD output. (C) 2001 Elsevier Science B.V. All rights reserved.

Keywords: Computer-aided design; Input devices; Task analysis; Blackboard model; Human factors

\section{Introduction}

In performing computer-aided design (CAD) tasks, designers are often faced with alternative means of interacting with the computer. A wide range of input and output $(\mathrm{I} / \mathrm{O})$ devices are available, each with its own properties that influence the appropriateness for a given task or context. The tablet-plus-keyboard has been the most common configuration in CAD systems. The alphanumeric keyboard must be learned and requires typing skills. A graphics tablet with several menu overlays does not require pointing skills, but its use leads to a division of attention between the display and the tablet. Depending on the task, operating the keyboard and tablet 